\title{
Validation of the use of Actigraph GT3X accelerometers to estimate energy expenditure in full time manual wheelchair users with spinal cord injury
}

\author{
X García-Massó $^{1}$, P Serra-Añó ${ }^{1}$, LM García-Raffi ${ }^{2}$, EA Sánchez-Pérez ${ }^{2}$, J López-Pascual ${ }^{3}$ and LM Gonzalez ${ }^{4}$
}

\begin{abstract}
Study design: Cross-sectional validation study.
Objectives: The goals of this study were to validate the use of accelerometers by means of multiple linear models (MLMs) to estimate the $\mathrm{O}_{2}$ consumption $\left(\mathrm{VO}_{2}\right)$ in paraplegic persons and to determine the best placement for accelerometers on the human body.

Setting: Non-hospitalized paraplegics' community.

Methods: Twenty participants (age $=40.03$ years, weight $=75.8 \mathrm{~kg}$ and height $=1.76 \mathrm{~m}$ ) completed sedentary, propulsion and housework activities for $10 \mathrm{~min}$ each. A portable gas analyzer was used to record $\mathrm{VO}_{2}$. Additionally, four accelerometers (placed on the non-dominant chest, non-dominant waist and both wrists) were used to collect second-by-second acceleration signals. Minute-byminute $\mathrm{VO}_{2}\left(\mathrm{ml} \mathrm{kg}^{-1} \mathrm{~min}^{-1}\right)$ collected from minutes 4 to 7 was used as the dependent variable. Thirty-six features extracted from the acceleration signals were used as independent variables. These variables were, for each axis including the resultant vector, the percentiles 10th, 25th, 50th, 75th and 90th; the autocorrelation with lag of $1 \mathrm{~s}$ and three variables extracted from wavelet analysis. The independent variables that were determined to be statistically significant using the forward stepwise method were subsequently analyzed using MLMs.
\end{abstract}

Results: The model obtained for the non-dominant wrist was the most accurate $\left(\mathrm{VO}_{2}=4.0558-0.0318 Y_{25}+0.0107 Y_{90}+0.0051\right.$ $\mathrm{Y}_{\mathrm{ND2} 2}-0.0061 Z_{\mathrm{ND2}}+0.0357 \mathrm{VR}_{50}$ ) with an $r$-value of 0.86 and a root mean square error of $2.23 \mathrm{ml} \mathrm{kg} \mathrm{m} \mathrm{min}^{-1}$.

Conclusions: The use of MLMs is appropriate to estimate $\mathrm{VO}_{2}$ by accelerometer data in paraplegic persons. The model obtained to the non-dominant wrist accelerometer (best placement) data improves the previous models for this population.

Spinal Cord (2013) 51, 898-903; doi:10.1038/sc.2013.85; published online 3 September 2013

Keywords: paraplegia; physical activity; signal processing; accelerometer; evaluation methodology

\section{INTRODUCTION}

People with spinal cord injury (SCI) adopt sedentary habits as a consequence of their disability. ${ }^{1}$ Sedentary habits worsen fitness in persons with SCI compared with their able-bodied peers ${ }^{1}$ and, in some cases, these individuals present a higher risk of suffering from long-term disorders or malfunctions of their organs and systems. ${ }^{2}$

Physical activity (PA) protects against such malfunctions or pathologies ${ }^{3-5}$ and is inversely correlated with all-cause mortality. While most of the studies in the literature that have analyzed the relationship between PA and disease prevention have been conducted with able-bodied persons, there are a few epidemiological studies performed in persons with SCI that have shown similar results. ${ }^{6-9}$ For this reason, it is very important to know whether persons with SCI who perform a minimum level of PA can avoid disorders associated with a sedentary lifestyle.

To date, most of the studies using able-bodied persons have employed questionnaires to assess PA. This method is inexpensive and easy to administer. Nevertheless, questionnaires present greater subjectivity, and the results depend on the accuracy of the subjects' memories. ${ }^{10}$
Other methods employed to estimate the PA level based on energy expenditure are indirect calorimetry and heart rate monitors. ${ }^{10}$ Due to the high price and the difficulty of employing indirect calorimetry measures in a daily scenario and the low accuracy of heart rate monitoring (during group calibration), neither option is optimal for PA assessment. ${ }^{10}$

Another technology used to assess energy expenditure is accelerometry, which is inexpensive, accurate and could be employed in daily activity. ${ }^{10}$ In fact, this technique has been one of the most widely accepted method for assessing PA in recent decades and has been validated in numerous recent studies. ${ }^{10,11}$ Thanks to all these studies about the validation, now there are several papers where the PA in free-living conditions in able-bodied people is evaluated using accelerometers. ${ }^{12,13}$

In persons with SCI, only a few studies have focused on the relationship between accelerations and energy expenditure values. ${ }^{14-18}$ Broadly, these studies present some restrictions. For example, the equations were obtained for a restricted number of daily activities; and consequently, the estimation of the energy expenditure in a real scenario could be biased. ${ }^{19}$ Likewise, in most of these previous studies, the authors chose integration epochs of $1 \mathrm{~min}$, which

${ }^{1}$ Departamento de Fisioterapia, Universidad de Valencia, Valencia, Spain; ${ }^{2}$ Instituto Universitario de Matemática Pura y Aplicada, Universidad Politécnica de Valencia, Valencia, Spain; ${ }^{3}$ Instituto de Biomecánica de Valencia, Valencia, Spain and ${ }^{4}$ Departamento de Educación Física y Deportiva, Universidad de Valencia, Valencia, Spain 
implies having only one feature for the estimation of minute-tominute energy expenditure.

On the other hand, the placement location is a critical point to estimate energy expenditure from accelerometer. There are studies in persons with disabilities (for example, multiple sclerosis or chronic obstructive pulmonary disease) where the best placement location is studied. ${ }^{20,21}$ This topic should be investigated in spinal cord-injured people due to their restricted patterns of movements.

Therefore, the main goal of this study is to validate the use of accelerometers by means of multiple linear models (MLMs) to estimate the $\mathrm{O}_{2}$ consumption $\left(\mathrm{VO}_{2}\right)$ in paraplegic persons. Furthermore, this study also aims to determine the best placement of the accelerometer on the human body to obtain the best possible estimation.

\section{MATERIALS AND METHODS}

\section{Participants}

A consecutive non-randomized sample of 20 subjects whose age, weight and height, in mean (s.d.), were 40.03 (10.57) years, 75.8 (17.54) kg and $1.76(0.09) \mathrm{m}$, respectively, participated in the study. The participants were recruited from the Hospital la $\mathrm{Fe}$ of Valencia and from the Asociación Provincial de Lesionados Medulares y Grandes Discapacitados (ASPAYM). These subjects were selected using the following inclusion criteria: (i) spinal injury between T2 and L5 and diagnosed 1 year before beginning study participation, (ii) full time wheelchair users and (iii) completely lost motor ability in their lower extremities (50/100 in ASIA impairment scale). The cause of the injury was traumatic in fifteen of the participants, tumoral in two subjects, iatrogenic in one case, and multiple sclerosis and congenital sclerosis in two more cases.

Subjects were excluded if they presented depressive or cognitive disorders; suffered from posttraumatic cervical myelopathy, motor or sensory impairment of the upper extremities, ischemic heart disorder, or recent osteoporotic fractures; had been tracheotomized; or presented sacrotuberous ulcers or hypertension. All subjects gave written consent to participate in the study (approved by the ethical committee of the University of Valencia). We certify that all applicable institutional and governmental regulations concerning the ethical use of human volunteers were followed during the course of this research.

\section{Data collection}

All subjects completed a routine of 10 activities: lying down, body transfers, moving items, mopping, working on a computer, watching TV, arm-ergometer exercise, passive propulsion, slow propulsion and fast propulsion. These activities of daily living were selected with the objective of having a wide range of intensities of PA and being typical for manual wheelchair users (Table 1). The subjects carried out each activity for $10 \mathrm{~min}$ with 1-2 min of rest between activities. There was only one exception corresponding to the activity of body transfers. In this case, the subjects carried out the activity for $1 \mathrm{~min}$ and rested for another minute for a total of $10 \mathrm{~min}$. The transfer task was configured in this way to avoid an overload of the musculoskeletal system in the shoulders.

During each activity, $\mathrm{VO}_{2}$ was monitored with the Cosmed $\mathrm{K} 4 \mathrm{~b}^{2}$ portable (Cosmed, Rome, Italy) gas analysis system. The calibration and placement of the device took into account as per instructions provided by the manufacturer. This device has been broadly employed as criterion to validate accelerometers. Macfarlane ${ }^{22}$ published a manuscript about the validity and reliability of different systems to measure the $\mathrm{VO}_{2}$ where the readers can check these data for the Cosmed $\mathrm{K}_{4} \mathrm{~b}^{2}$. The subjects wore four accelerometers (Actigraph model GT3X; Actigraph, Pensacola, FL, USA): one on each wrist, one on the waist (above the non-dominant anterior superior iliac crest) and the last in the chest (below the non-dominant armpit at the height of the xiphoid apophysis) (Figure 1). The Actigraph was initialized using 1-s epochs, and the time was synchronized with a digital clock so the start time could be synchronized with the gas analyzer.

\section{Signal processing}

The Matlab R2010a (Mathworks Inc, Natick, MA, USA) program was used for preprocessing, segmentation and feature extraction from the signals. The $\mathrm{VO}_{2}$ signal was preprocessed using averaged blocks of $30 \mathrm{~s}$. The time interval between the start of minute 4 and the end of minute 7 was selected. The $\mathrm{VO}_{2}$ expressed in $\mathrm{ml} \mathrm{kg}^{-1} \mathrm{~min}^{-1}$ was calculated for each of these minutes. The segmentation of the signals was similar to previous works and confirmed that steady-state $\mathrm{VO}_{2}$ was reached. ${ }^{23}$ The $\mathrm{VO}_{2}$ for each of the selected minutes was used as the dependent or output variable in the designed models.

The outputs from accelerometers (counts $\mathrm{s}^{-1}$ ) were used to obtain predicting variables. Counts are used extensively in this topic and they represent the amount of acceleration between two consecutive levels of quantization during the analogical-to-digital conversion. We obtained nine total variables for each axis (that is, $\mathrm{X}, \mathrm{Y}, \mathrm{Z}$ and resultant vector) in minutes number four, five, six and seven of each activity. These variables correspond to features that have been extracted from the time domain and from the discrete wavelet transform (DWT) of the signal. In the time domain, the 10th, 25th, 50th, 75th and 90th percentiles were calculated. Furthermore, as a measurement of the temporal dynamics, the lag-one correlation of each minute was calculated. ${ }^{23}$

Finally, three variables were included as a result of the DWT. To present the experimental information in a compressed and arranged format, we have analyzed the signal with multiresolution analysis based on wavelet transform. ${ }^{24,25}$ The signal was sampled up to two levels of decomposition using the Daubechies 2 mother wavelet. We calculated the Euclidean norm of the three vectors corresponding to the detail coefficients of the first and second levels of resolution and the approximation coefficients of the second level (that is, $\mathrm{ND}_{1}$, $\mathrm{ND}_{2}$, and $\mathrm{NA}_{2}$ ). These variables were also included in our analysis (all the descriptive parameters can be seen in the Supplementary File).

\section{Mathematical models}

We obtained an MLM for each placement location. We only used statistically significant features determined by the forward stepwise method.

\section{Table 1 Activity routine}

\begin{tabular}{lll}
\hline Order & Activity & Description \\
\hline 1 & Lying down & Lying in the lateral decubitus position \\
2 & Body transfers & Self-shifting the bodyweight from one side to the other using a stretcher (simulating body transfers) \\
3 & Moving items & Loading and transferring boxes with different weights between shelves placed on opposite sides of the laboratory \\
4 & Mopping & Simulation of mopping housework throughout the laboratory \\
5 & Watching TV & Viewing of different television programs \\
6 & Working on a computer & Simulation of personal computer work using a word processing program and the internet \\
7 & Arm-ergometry exercise & Performance of an ergometer work sequence with an intensity corresponding to a perception of eight points based on \\
8 & the OMNI-Res perception scale \\
9 & Passive propulsion & Propulsion of the individual by the researcher \\
10 & Fast propulsion & Self-propulsion of the wheelchair over the floor at a moderate speed \\
\end{tabular}




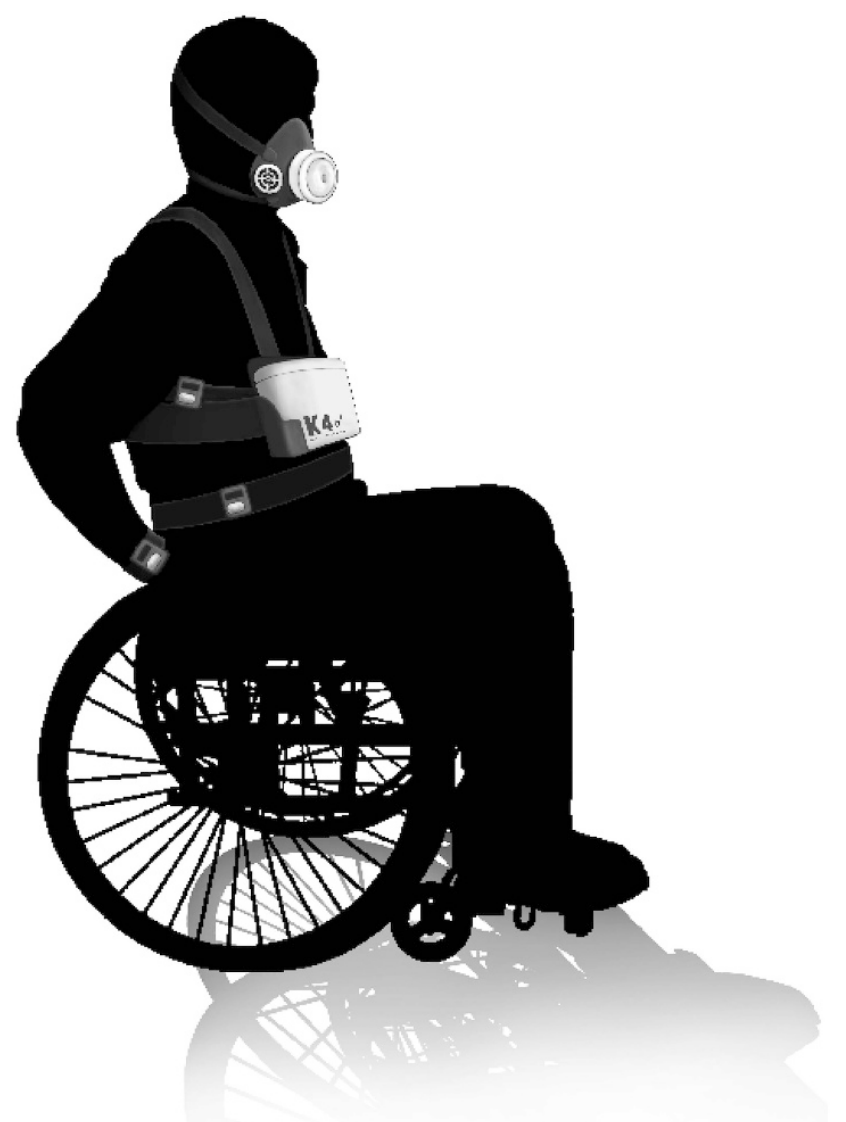

Figure 1 Instrumentation set-up. The individual wear four accelerometers: one on each wrist, one on the waist and one in the chest. Further, the portable gas analysis system is placed with a harness on the chest and connected to the mask, placed over the mouth and nose.

The dependent variable was the consumption of $\mathrm{VO}_{2}$ in every minute (that is, 800 values in total). The validation of the model was determined by 20 -fold cross-validation. For every model, we computed the following statistical parameters: mean square error (MSE), mean absolute error (MAE), root mean square error (RMSE) and the coefficient of correlation $(r)$. Moreover, we calculated the mean error and the percentage error between the estimation and the $\mathrm{VO}_{2}$ measured by $\mathrm{K} \mathrm{b}^{2}$ for the validation data. The Student's test for related samples was performed to establish significant differences between criterion and estimate $\mathrm{VO}_{2}$ values. The level of significance was set at $P=0.05$.

\section{RESULTS}

From the analysis of our data, we obtained four linear models with multiple independent variables, one model for each placement location. The models for the waist and the chest have 18 and 11 independent variables, respectively. Due to the large number of independent variables and poor performance of the waist and chest models compared with those corresponding to each wrist, these equations have been included in the Supplementary File. Model 1 (equation 1) corresponds to the data obtained from the dominant wrist, whereas model 2 (equation 2) corresponds to the data obtained from the non-dominant wrist.

$$
\begin{aligned}
\mathrm{VO}_{2}= & 4.1355+0.0376 X_{50}-0.0155 X_{90}-0.0047 X_{\mathrm{NA}_{1}} \\
+ & 0.0062 X_{\mathrm{ND}_{1}}+0.02 Z_{75}-0.0363 Z_{90}+0.0161 \mathrm{VR}_{75}+0.253 \mathrm{VR}_{90} \\
\mathrm{VO}_{2}= & 4.0558-0.0318 Y_{25}+0.0107 Y_{90} \\
& +0.0051 Y_{\mathrm{ND}_{2}}-0.0061 Z_{\mathrm{ND}_{2}}+0.0357 \mathrm{VR}_{50}
\end{aligned}
$$

Table 2 General linear model efficiency of the four accelerometers

\begin{tabular}{llllll}
\hline Location & Data & $r$ & MSE & MAE & RMSE \\
\hline Waist & Fit & 0.64 & 11.33 & 2.47 & 3.32 \\
& Validation & 0.67 & 10.61 & 2.39 & 3.26 \\
\multirow{4}{*}{ Chest } & All & 0.67 & 10.65 & 2.39 & 3.26 \\
& Fit & 0.66 & 10.80 & 2.45 & 3.26 \\
& Validation & 0.68 & 10.41 & 2.41 & 3.23 \\
Dominant wrist & All & 0.68 & 10.43 & 2.41 & 3.23 \\
& Fit & 0.85 & 5.32 & 1.69 & 2.28 \\
& Validation & 0.86 & 5.16 & 1.67 & 2.27 \\
Non-dominant wrist & All & 0.86 & 5.16 & 1.67 & 2.27 \\
& Fit & 0.86 & 5.08 & 1.66 & 2.23 \\
& Validation & 0.86 & 4.98 & 1.65 & 2.23 \\
& All & 0.86 & 4.98 & 1.65 & 2.23 \\
\hline
\end{tabular}

Abbreviations: MAE, mean absolute error; MSE, mean square error; $r$, coefficient of correlation; $\mathrm{RMSE}=$ root mean square error.

The label 'Fit' in the table corresponds to the data set used to fit the model. The label

'Validation' corresponds to the data set used to validate the model. Finally, the label 'All' corresponds to fit and validation data sets together.

in these equations, capital letters $X, Y$ and $Z$ represent axes, the sub-indexes represent variables and VR is the resultant vector. The sub-indexes 25, 50, 75 and 90 are percentiles, and for the variable $J$, the symbol $J_{i}$ for $i=25,50,75$ and 90 denotes the value of the ith percentile of the variable $J$. The norm of the vector of the approximation coefficients in the first level in DWT is denoted by $\mathrm{NA}_{1}$, the norm of the vector of the detail coefficients in the first level by $\mathrm{ND}_{1}$, and the norm of the vector of the detail coefficients in the second level by $\mathrm{ND}_{2}$. It can be noted that equation 2 has five independent variables, whereas equation 1 has eight.

The models corresponding to both wrists provide a good estimate of $\mathrm{VO}_{2}$. The predictions obtained using the accelerometers corresponding to the chest and waist were much less accurate (Table 2).

In Figure 2, we show dispersion and Bland-Altman plots for each of the models established. In each case analyzed, no systematic error is observed, but the residuals obtained in the models for the waist and the chest are large (that is, wider range between \pm 2 s.d.).

Additionally, in each of the Bland-Altman plots, there is a tendency to underestimate $\mathrm{VO}_{2}$ for values larger than $20 \mathrm{ml} \mathrm{kg}^{-1} \mathrm{~min}^{-1}$. This tendency is less pronounced for the model corresponding to the nondominant wrist. Moreover, when we analyzed the error in each activity expressed as a percent, the relative values obtained were all lower than $20 \%$ for both models of the dominant and non-dominant wrist (Table 3). Both the mean (s.d.) of the $\mathrm{VO}_{2}$ measured with $\mathrm{K}_{4} \mathrm{~b}^{2}$ and the values estimated for each accelerometer in each activity are shown in Table 4.

\section{DISCUSSION}

The fitting models obtained in the present study improve the data previously published related to the assessment of PA in paraplegic subjects by means of accelerometry. This improvement can be seen in both the achievement of a stronger correlation between the estimation of $\mathrm{VO}_{2}$ and the measured value and a lower prediction error for the activities evaluated. We interpret these data to be the result of our use of 1-s epochs for the acquisition of acceleration data.

To the best of our knowledge, there are few studies that have estimated the energy expenditure in persons with paraplegia by means of movement sensors, and most of these studies have used 1-min epochs of accelerometry data. ${ }^{14-18}$ The current study aimed to improve this aspect by including statistical parameters about count distribution during each minute through the acquisition of 1-s epochs. Due to this 

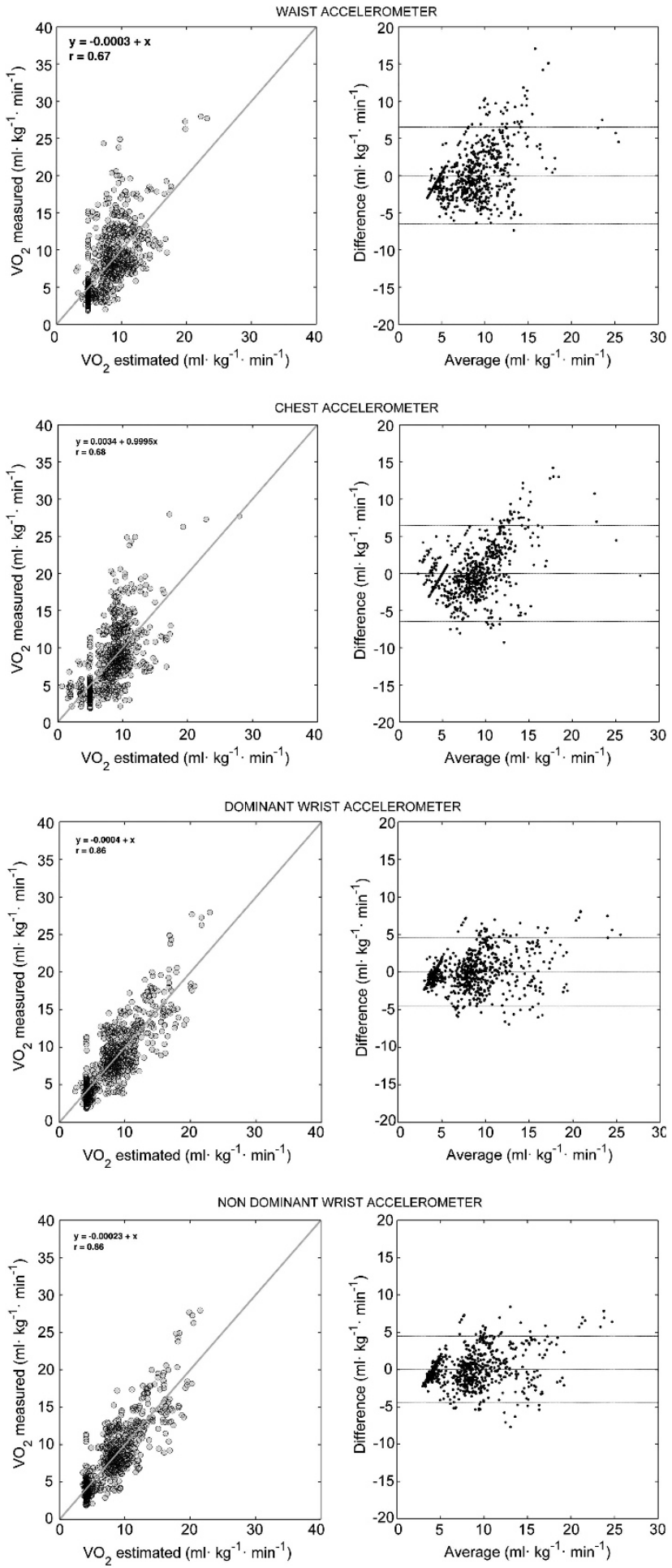

Figure 2 Scatter and Bland-Altman plots of the general linear models. The 800 data points represent the values of the consumption of $\mathrm{VO}_{2}$ in every minute of each participant performing all the activities. The left panel displays the scatter plots of the real value against the estimated value. The gray line represents the regression line between both values. The right panel displays the Bland-Altman plots. The discontinuous lines represent the means of the residuals and the means \pm 2 s.d.

amount of data (60 per minute), we can perform a feature extraction process and, as a consequence, obtain several variables with relevant information for the estimation of the energetic expenditure.
Moreover, performing 10 different tasks that are representative of daily living provides a wide variety of motion patterns. This variety gives greater consistency to the estimation method obtained. Previous studies only performed sedentary tasks, propulsion by wheelchair and arm-ergometer exercise. Therefore, the estimation methods employed could be insufficient for assessing of different motion patterns (for example, transfers and mopping). ${ }^{14-18}$

Among the models generated in our study, those of the wrists are more accurate than the rest as it can be concluded from the values of their MAE, MSE, RMSE and Pearson's coefficients. Moreover, the percentage error for each activity was lower for wrist models than for chest and waist equations. This can be due to the reduced mobility of the chest and waist of people with SCI. This fact could produce that the accelerations in these locations and the corresponding intensity of the activity were not well correlated or even uncorrelated.

Regarding the $\mathrm{VO}_{2}$ values obtained with the gas analyzer from the participants performing the tasks, the data were confirmed to be similar to those provided in previous studies. In the slow propulsion task, the consumption measured in our work (that is, $7.42 \mathrm{ml} \mathrm{kg}^{-1} \mathrm{~min}^{-1}$ ) was almost identical to previous values reported (that is, 7.35-7.4) when the task was executed at a rate of $4.8-4.9 \mathrm{~km} \mathrm{~h}^{-1} .14,16$

Similar results were also observed in previous studies for other tasks, such as working on a computer, ${ }^{14,26,27}$ watching $\mathrm{TV}^{27}$ and moving items. ${ }^{26,27}$ Regarding arm-ergometer exercise, we obtained a value of $14.83 \mathrm{ml} \mathrm{kg}^{-1} \mathrm{~min}^{-1}$, and we have found values from 7.66 to $20.55 \mathrm{ml} \mathrm{kg}^{-1} \mathrm{~min}^{-1}$ in the previous literature, depending on the power developed and the level of the SCI. ${ }^{14,26,27}$

The first paper that tried to establish regression equations to estimate the $\mathrm{VO}_{2}$ in persons with SCI through accelerometry was written by Washburn and Copay in $1999 .{ }^{16}$ They obtained a simple linear equation using the accelerations of the non-dominant wrist with an standard error of the estimation of $4.99 \mathrm{ml} \mathrm{kg}^{-1} \mathrm{~min}^{-1}$. Furthermore, they could explain $44 \%$ of the variability of the $\mathrm{VO}_{2}$ using the counts $\min ^{-1}$. Comparing these results with those obtained with the general linear model employed in our study, we can observe some improvements. It is important to note that the estimation errors and the $r$-value depend on the number and type of activities performed to acquire the data used in the validation. Nevertheless to compare between estimators we only have these parameters as they are commonly reported in the validation studies. The RMSE in our work is $2.23 \mathrm{ml} \mathrm{kg}^{-1} \mathrm{~min}^{-1}$, and the determination coefficient has a value of 0.74 . In view of these results, we found that the use of methodologies that maximize the data available for the estimation of $\mathrm{VO}_{2}$ can provide general linear models that have better accuracy.

Recently, Hiremath and Ding ${ }^{17}$ developed a new equation based on an MLM that was designed with 19 individuals and tested on another 4 for validation. Acceleration data were obtained from the left arm, and indirect calorimetry was employed as a reference measurement during the performance of a limited routine activities. With the data used to develop the equation (the fitting data set), the authors found an standard error of the estimation of $1.02 \mathrm{kcal} \mathrm{min}^{-1}$ (2.55 $\mathrm{ml} \mathrm{kg}^{-1} \mathrm{~min}^{-1}$ approximately) and an $r^{2}$ of 0.7 . Although these authors improved preexisting models, the estimation was not as accurate as those models for persons without disabilities. This discrepancy was due to the considerable percentage of error observed for the validation data; this error ranged from $14.12 \%$ for armergometer exercise (at $40 \mathrm{~W}$ and 90 r.p.m.) up to $113.68 \%$ for the resting task.

The MLM of the non-dominant wrist designed in our study has shown values of RMSE and $r^{2}$ similar to those obtained in the previous studies. However, the percentage of error in each of the 
Table 3 Errors in each of the activities obtained with the general linear models in the validation subset

\begin{tabular}{|c|c|c|c|c|}
\hline Activity & Waist & Chest & Dominant wrist & Non-dominant wrist \\
\hline Lying down & $31.86 \%(1.21 ; 0.98-1.45)$ & $26.09 \%(1.01 ; 0.77-1.24)$ & $10.51 \%(0.4 ; 0.2-0.6)$ & $9.55 \%(0.37 ; 0.15-0.58)$ \\
\hline Body transfers & $5.14 \%(0.54 ;-0.18-1.27)$ & $10.11 \%(1.05 ; 0.39-1.72)$ & $\begin{array}{c}18.42 \%(-1.86 ;-2.29 \\
\text { to }-1.43)\end{array}$ & $\begin{array}{c}18.68 \%(-1.87 ;-2.37 \\
\text { to }-1.36)\end{array}$ \\
\hline Moving items & $5.63 \%(-0.53 ;-1.13-0.07)$ & $\begin{array}{c}6.76 \%(-0.65 ;-1.25 \\
\text { to }-0.05)\end{array}$ & $4.80 \%(-0.46 ;-0.94-0.02)$ & $0.67 \%(-0.06 ;-0.5-0.37)$ \\
\hline Mopping & $15.71 \%(1.23 ; 0.74-1.72)$ & $10.39 \%(0.82 ; 0.34-1.3)$ & $12.35 \%(0.98 ; 0.49-1.47)$ & $6.85 \%(0.54 ; 0.09-0.99)$ \\
\hline Arm-ergometry exercise & $\begin{array}{c}31.51 \%(-4.69 ;-5.69 \\
\text { to }-3.39)\end{array}$ & $\begin{array}{c}24.77 \%(-3.69 ;-4.62 \\
\text { to }-2.76)\end{array}$ & $0.81 \%(0.13 ;-0.64-0.9)$ & $1.11 \%(0.16 ;-0.65-0.97)$ \\
\hline Passive propulsion & $39.27 \%(1.66 ; 1.31-2.0)$ & $30.74 \%(1.29 ; 0.85-1.74)$ & $7.70 \%(0.32 ;-0.009-0.65)$ & $6.30 \%(0.27 ;-0.03-0.57)$ \\
\hline Slow propulsion & $12.51 \%(0.93 ; 0.48-1.38)$ & $11.62 \%(0.88 ; 0.33-1.43)$ & $9.81 \%(0.72 ; 0.32-1.13)$ & $12.67 \%(0.93 ; 0.55-1.32)$ \\
\hline Fast propulsion & $\begin{array}{c}22.29 \%(-2.96 ;-3.84 \\
\text { to }-2.09)\end{array}$ & $\begin{array}{c}28.33 \%(-3.74 ;-4.5 \\
\text { to }-2.96)\end{array}$ & $\begin{array}{c}9.31 \%(-1.21 ;-1.96 \\
\text { to }-0.45)\end{array}$ & $\begin{array}{c}7.90 \%(-1.06 ;-1.73 \\
\text { to }-0.38)\end{array}$ \\
\hline
\end{tabular}

Data between brackets are the mean differences between real and estimated oxygen consumption $\left(\mathrm{ml} \mathrm{kg}^{-1} \mathrm{~min}^{-1}\right)$ and the confidence intervals.

Table 4 Measured and estimate $\mathrm{VO}_{2}$ for validation data

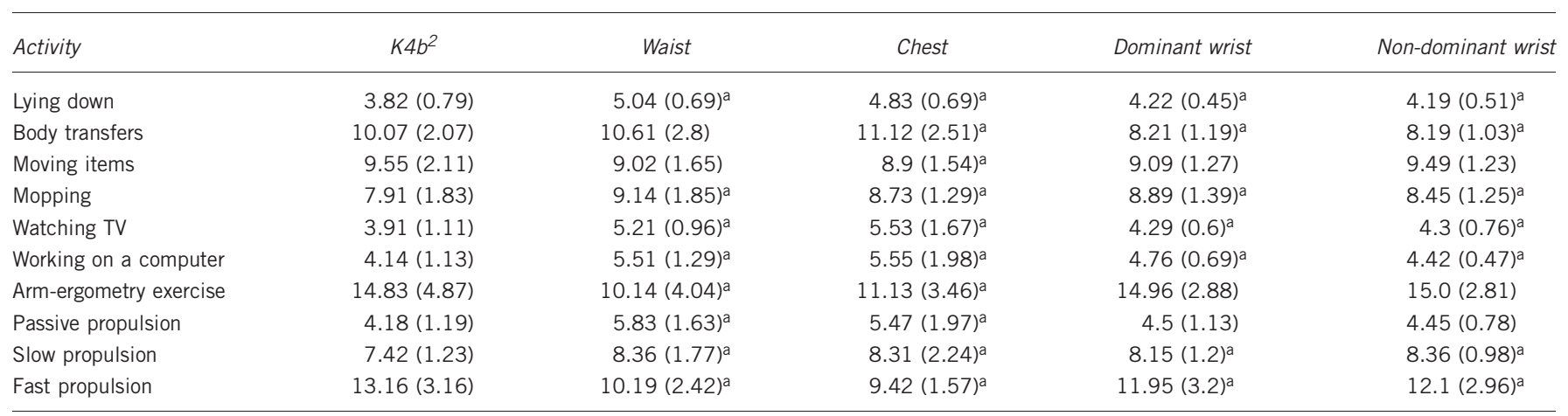

Data are expressed as mean (s.d.).

andicates significant differences $(P<0.05)$ with respect $k 4 \mathrm{~b}^{2}$ value.

activities is lower, and there is less dispersion between activities. Moreover, the minimum and maximum error obtained was $0.67 \%$ and $18.68 \%$, respectively. For this reason, the MLM applied in this study provides a methodological improvement for the prediction of the $\mathrm{VO}_{2}$ in persons with SCI. In our case, the model for persons with paraplegia showed similar estimation errors than the models corresponding to persons without disabilities such as the two-regression model $^{28}$ or artificial neural network-based models ${ }^{23}$ (although these models were designed with more activities than our model).

The present study does have some limitations. First, although the participants performed a wide range of activities, there are additional activities that should be assessed in future studies (for example, sports activities as basketball or household activities as washing dishes). Due to the difficulty in recruiting individuals with SCI and the significant administrative burden in the application of all of the protocols, it was not possible to extend the number of tasks executed. In this sense, it could be also interesting to increase the number of subjects for considering a wider inter-subjects variability and, therefore, to improve the robustness of the estimator. Acceleration data have been recorded in counts $\mathrm{s}^{-1}$; raw acceleration data in $\mathrm{m} \mathrm{s}^{-2}$ would provide more information and therefore a more accurate estimation. Nevertheless, we chose 1-s epochs because of the memory limitation of the GT3X.
In conclusion, MLM that employ feature extraction from accelerometer signals measured in counts $\mathrm{s}^{-1}$ can be used to obtain accurate estimations of the $\mathrm{VO}_{2}$ in paraplegic persons. Furthermore, it has been demonstrated that in this population, it is possible to record data from either wrist, although there are some benefits of using the non-dominant wrist (that is, fewer predictive variables and slightly better parameters of performance).

The results of our study could be used to understand the PA level in SCI and guide future descriptive studies in this population. The results presented in this work can contribute to identify patients who are at risk of suffering from problems related to a sedentary lifestyle.

\section{DATA ARCHIVING}

There were no data to deposit.

\section{CONFLICT OF INTEREST}

The authors declare no conflict of interest.

\section{ACKNOWLEDGEMENTS}

LM García-Raffi and EA Sánchez-Pérez gratefully acknowledge the support of the Ministerio de Economía y Competitividad under project \#MTM201236740-c02-02. X García-Massó is a Vali + D researcher in training with support from the Generalitat Valenciana. 
1 Van den Berg-Emons RJ, Bussmann JB, Haisma JA, Sluis TA, van der Woude LH, Bergen MP et al. A prospective study on physical activity levels after spinal cord injury during inpatient rehabilitation and the year after discharge. Arch Phys Med Rehabil 2008; 89: 2094-2101.

2 Jacobs PL, Nash MS. Exercise recommendations for individuals with spinal cord injury. Sports Med 2004; 34: 727-751.

3 Erikssen G. Physical fitness and changes in mortality: the survival of the fittest. Sports Med 2001; 31: 571-576.

4 Warburton DER, Nicol CW, Bredin SSD. Health benefits of physical activity: the evidence. CMAJ 2006; 174: 801-809.

5 Haennel RG, Lemire F. Physical activity to prevent cardiovascular disease. How much is enough? Can Fam Physician 2002; 48: 65-71.

6 Manns PJ, Chad KE. Determining the relation between quality of life, handicap, fitness, and physical activity for persons with spinal cord injury. Arch Phys Med Rehabil 1999; 80: 1566-1571.

7 Hetz SP, Latimer AE, Buchholz AC, Martin Ginis KA. Increased participation in activities of daily living is associated with lower cholesterol levels in people with spinal cord injury. Arch Phys Med Rehabil 2009; 90: 1755-1759.

8 Buchholz AC, Martin Ginis KA, Bray SR, Craven BC, Hicks AL, Hayes KC et al. Greater daily leisure time physical activity is associated with lower chronic disease risk in adults with spinal cord injury. Appl Physiol Nutr Metab 2009; 34: 640-647.

9 Slater D, Meade MA. Participation in recreation and sports for persons with spinal cord injury: review and recommendations. Neurorehabilitation 2004; 19: 121-129.

10 Valanou EM, Bamia C, Trichopoulou A. Methodology of physical-activity and energyexpenditure assessment: a review. J Public Health 2006; 14: 58-65.

11 Liu S, Gao RX, Freedson PS. Computational methods for estimating energy expenditure in human physical activities. Med Sci Sports Exerc 2012; 44: 2138-2146.

12 Troiano RP, Berrigan D, Dodd KW, Mâsse LC, Tilert T, McDowell M. Physical activity in the United States measured by accelerometer. Med Sci Sports Exerc 2008; 40: 181-188.

13 Riddoch CJ, Bo Andersen L, Wedderkopp N, Harro M, Klasson-Heggebø L, Sardinha LB et al. Physical activity levels and patterns of 9- and 15-yr-old European children. Med Sci Sports Exerc 2004; 36: 86-92.

14 Hiremath SV, Ding D. Evaluation of activity monitors in manual wheelchair users with paraplegia. J Spinal Cord Med 2011; 34: 110-117.
15 Hiremath SV, Ding D. Evaluation of activity monitors to estimate energy expenditure in manual wheelchair users. Conf Proc IEEE Eng Med Biol Soc 2009; 2009: 835-838.

16 Washburn R, Copay A. Assessing physical activity during wheelchair pushing: validity of a portable accelerometer. Adapt Phys Activ Q 1999; 16: 290-299.

17 Hiremath SV, Ding D. Regression equations for RT3 activity monitors to estimate energy expenditure in manual wheelchair users. Conf Proc IEEE Eng Med Biol Soc 2011; 2011: 7348-7351.

18 Hiremath SV, Ding D, Farringdon J, Cooper RA. Predicting energy expenditure of manual wheelchair users with spinal cord injury using a multisensor-based activity monitor. Arch Phys Med Rehabil 2012; 93: 1937-1943.

19 Bassett DR Jr, Ainsworth BE, Swartz AM, Strath SJ, O'Brien WL, King GA. Validity of four motion sensors in measuring moderate intensity physical activity. Med Sci Sports Exerc 2000; 32: S471-S480.

20 Motl RW, Sosnoff JJ, Dlugonski D, Suh Y, Goldman M. Does a waist-worn accelerometer capture intra- and inter-person variation in walking behavior among persons with multiple sclerosis? Med Eng Phys 2010; 32: 1224-1228.

21 Van Remoortel H, Raste Y, Louvaris Z, Giavedoni S, Burtin C, Langer D et al. Validity of six activity monitors in chronic obstructive pulmonary disease: a comparison with indirect calorimetry. PLoS One 2012; 7: e39198.

22 Macfarlane DJ. Automated metabolic gas analysis systems: a review. Sports Med $2001 ; 31: 841-861$.

23 Staudenmayer J, Pober D, Crouter S, Bassett D, Freedson P. An artificial neural network to estimate physical activity energy expenditure and identify physical activity type from an accelerometer. J Appl Physiol 2009; 107: 1300-1307.

24 Daubechies I. Ten Lectures on Wavelets. SIAM, Philadelphia, 1999.

25 Debnat I. Wavelets and Signal Processing. Birkhauser, Boston, 2003.

26 Collins EG, Gater D, Kiratli J, Butler J, Hanson K, Langbein WE. Energy cost of physical activities in persons with spinal cord injury. Med Sci Sports Exerc 2010; 42: 691-700.

27 Lee M, Zhu W, Hedrick B, Fernhall B. Determining metabolic equivalent values of physical activities for persons with paraplegia. Disabil Rehabil 2010; 32: 336-343.

28 Crouter SE, Clowers KG, Bassett DR Jr. A novel method for using accelerometer data to predict energy expenditure. J Appl Physiol 2006; 100: 1324-1331.

Supplementary Information accompanies this paper on the Spinal Cord website (http://www.nature.com/sc) 